POLLACK PERIODICA

An International Journal for Engineering and Information Sciences

DOI: $10.1556 / 606.2017 .12 .1 .3$

Vol. 12, No. 1, pp. 29-44 (2017)

www.akademiai.com

\title{
NUMERICAL SOLUTION OF THE RICHARDS EQUATION BASED CATCHMENT RUNOFF MODEL WITH DD-ADAPTIVITY ALGORITHM AND BOUSSINESQ EQUATION ESTIMATOR
}

\author{
${ }^{1}$ Michal KURAZ, ${ }^{2}$ Jiri HOLUB, ${ }^{3}$ Jakub JERABEK \\ ${ }^{1,2,3}$ Faculty of Environmental Sciences, Czech University of Life Sciences \\ Prague, Kamycka 1176, 16521 Prague 6, Czech Republic \\ e-mail: ${ }^{1}$ kuraz@fzp.czu.cz, ${ }^{2}$ jholub@fzp.czu.cz, ${ }^{3}$ jerabek@fzp.czu.cz
}

Received 22 May 2016; accepted 13 December 2016

\begin{abstract}
This paper presents a pseudo-deterministic catchment runoff model based on the Richards equation model - the governing equation for subsurface flow. The subsurface flow in a catchment is described here by two-dimensional variably saturated flow (unsaturated and saturated). The governing equation is the Richards equation with a slight modification of the time derivative term, as considered e.g. by Neuman. The nonlinear nature of this problem appears in the unsaturated zone only, so it was possible to make use of adaptive domain decomposition algorithm. However delineating of the saturated zone boundary is a nonlinear computationally expensive issue. The simple one-dimensional Boussinesq equation was used here as a rough estimator of the saturated zone boundary. With this estimate the adaptive domain decomposition could always start with an optimal subdomain split, and thus it is now possible to avoid solving huge systems of linear equations in the initial iteration level.

With this measure it is possible to construct an efficient two-dimensional pseudodeterministic catchment runoff model. Finally, the model is tested against real data originating from the Modrava 2 experimental catchment, Czech Republic.
\end{abstract}

Keywords: Computational hydrology, Richards equation, Two-dimensional catchment runoff modeling, Domain decomposition method

\section{Introduction}

Runoff modeling forms a fundamental part of hydrology. It is an important tool for predicting the response of a catchment to extreme hydrological events like droughts or extreme rainfalls. For sustainable landscape development, it is essential to understand the behavior of the catchment. 
A distinction is often made between empirical and physically-based runoff models. Physically-based models compared to the empirical models can help the researcher to understand the processes that generate a discharge or lead to a specific soil moisture distribution. Particularly in steep forested catchments, rapid shallow subsurface runoff takes place in the absence of surface runoff [1], [2].

To model the transient soil moisture distribution at hillslope scale, Freeze [3] developed a very physically-based three-dimensional model describing variably saturated transient water flow in a porous medium.

It is not always necessary to perform three-dimensional computation. There have already been attempts to approximate the soil profile within a hillslope by a representative two-dimensional cross-section. For example, Kao et al. [4] used a twodimensional solution of the Richards' equation to explore the thickness of the transition zone above the water table and the flow direction in the transition zone. Assigning different hydraulic conductivities and solving the Richards' equation in a twodimensional profile to simulate an effect of soil pipes showed a satisfactory match with the measured data, see [5]. Another application can be found in [6], where the hillslope runoff regime was investigated on a two-dimensional model. Two-dimensional catchment runoff modeling is a classical but still state of the art issue. A hypothesis whether a single hillslope can represent entire mesoscale catchment was recently examined by Loritz et al. [7]. Another works by Bishop et al. [8], Wienhöfer and Zehe [9], and Klaus and Zehe [10] focused on the lateral and preferential flow networks in catchment by evaluating a physically based runoff model.

Modeling the hillslope discharge with the Boussinesq equation has been presented e.g. by Troch et al. [11] and Paniconi et al. [12]. This concept was tested on hillslopes with different shapes and slopes, while comparing the solution with the solution to the three-dimensional Richards equation.

An accurate and efficient numerical solution of the Richards equation has been worked on by several researchers. Time adaptivity algorithms were studied in Authors' papers [13], [14], while spatial adaptivity (h- and hp-adaptivity and adaptive domain decomposition (dd-adaptivity)) have been studied in other papers by the Authors [15], [16], [17], [18].

The aim of this paper is to present a numerical technique for solving the Richards equation in two-dimensions under variably saturated conditions (saturated and unsaturated), that combines the adaptive domain decomposition method (dd-adaptivity) and Boussinesq equation as an estimator. When solving the Richards equation the computational issue appears in the unsaturated zone, in the saturated zone the Richards equation degenerates into the Laplace equation, and so using the adaptive domain decomposition could significantly decrease the number of degrees of freedom of the nonlinear problem. The Bousinesq equation was used here for the initial subdomain split estimate. This two-dimensional Richards equation model with Boussinesq equation estimator was considered here as a catchment runoff model, which was finally tested on real-world case study - a seasonal runoff model of Modrava 2 experimental catchment, Czech Republic.

This paper is organized as follows. The governing equations for subsurface flow both the Richards equation and Boussinesq equation - are presented. The dd-adaptivity is briefly presented here, and a comparison is made with the previous works of the 
Author. The dd-adaptivity algorithm is combined here with the Boussinesq equation, which is used as a predictor for the initial subdomain split and the initial condition setup. The problem is implemented for a two-dimensional problem, where the catchment runoff is simulated as the runoff on a representative two-dimensional cross section. In order to provide an evaluation of the physically based concept, the model is tested against real hydrological data originating from Modrava experimental catchment, Czech Republic.

\section{Governing equations for subsurface flow}

The governing equations for subsurface flow originate from the law of mass conservation. Under saturated conditions, the flux is governed by the Darcy law. In case of variable saturation (both saturated and unsaturated), the flux is governed by the Darcy-Buckingham law, which can be understood as an extension of the Darcy law.

While the flow in a porous medium is naturally three-dimensional, and threedimensional problems produce difficulties in both numerical and analytical solutions, a number of approximations have been published in the past that reduce the dimensions of the problem.

The following sections will present the standard Richards equation, which can be used to describe both unsaturated flow and saturated flow in a porous medium. Later, the Boussinesq equation, originating from the well-known Dupuit approximation will be presented here. The Boussinesq equation approximates the naturally two-dimensional problem of the flow over a sloping impermeable layer into a one-dimensional problem. And finally the numerical solution of the Richards equation, which is used here as a runoff model, is presented in these sections. The Richards equation is efficiently treated here by $d d$-adaptivity [16], [17], [18], with the Boussinesq equation as a predictor.

\subsection{Richards equation}

In brief, the mathematical model of variably saturated flow (the saturated zone and the unsaturated zone) is assumed here as the Richards equation in $h$-form, where $h$ is a so-called pore hydrostatic pressure head $[L]$. This equation was originally published by L.A. Richards [19]. Modification of the original equation, which extends its validity into the saturated zone with non-zero specific storage, see e.g. Huyakorn [20] or Neuman [21] is considered here. Works by Tocci et al. [22] and also by Kuraz et al. [13], [14] suggest temporal adaptivities that preserve the mass balance.

The Richards equation in $h$-form is presumed as

$$
\nabla \cdot(\mathbf{K}(h) \nabla h)+\nabla \cdot\left(\mathbf{K}_{(t, n)}(h)\right)=C(h) \frac{\partial h}{\partial t}, \quad(x, t) \in[0, T)
$$

where $h$ is the capillary pressure head function [L]; $\mathbf{K}(h)$ is the unsaturated hydraulic

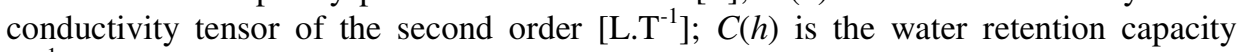
$\left[\mathrm{L}^{-1}\right]$ is usually defined as 


$$
C(h)=\frac{d \theta}{d h}+\frac{\theta(h)}{\theta_{S}} S_{S},
$$

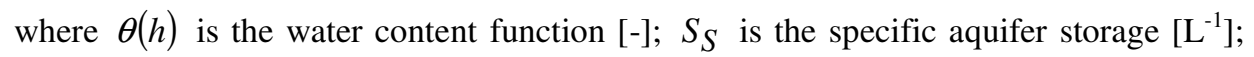
$\theta_{S}$ is the saturated water content [-]; and $\mathbf{K}_{(t, n)}(h)$ is a vector formed out of the last column of the hydraulic conductivity tensor, where index $n$ denotes the dimension of $\mathfrak{R}^{n}$, - this is a flux vector originating from gravitational forces. Constitutive relations for the function $\theta(h)$ and $\mathbf{K}(h)$ are supplied by van Genuchten's law [23] and by the Mualem model [24].

It is apparent that in the saturated state (for $h>0$ ) and for the homogeneous medium the equation (1) becomes

$$
S_{S} \frac{\partial h}{\partial t}=K_{S} \Delta h
$$

because if $h>0$ then $d \boldsymbol{\theta} / d h=0$; and $\mathbf{K}(h)$ becomes scalar $K(h)$, and $K(h)=K_{S}$ where $K_{S}$ is the saturated hydraulic conductivity (a constant) [L.T ${ }^{-1}$; and since the specific storage is often neglected $\left(S_{S}=0\right)$, the governing equation for the saturated state degenerates into a simple Laplace equation

$$
\Delta h=0 .
$$

It is apparent that $d d$-adaptivity algorithm will be highly efficient here.

\subsection{Boussinesq equation for a sloping impermeable layer}

The problem of saturated flow over a sloping impermeable layer can be treated by an extended form of the Dupuit-Forchheimer assumption, where the streamlines are parallel to the impermeable layer [25]. Then the Boussinesq equation states as follows [26]

$$
\frac{\theta_{S}}{K_{S} \cos \alpha} \frac{\partial h_{b}}{\partial t}=\frac{q(t)}{K_{S}}\left(1+\tan \alpha \frac{\partial h_{b}}{\partial x}\right)+\frac{\partial}{\partial x}\left(h_{b} \frac{\partial h_{b}}{\partial x}\right)-\tan \alpha \frac{\partial h_{b}}{\partial x},
$$

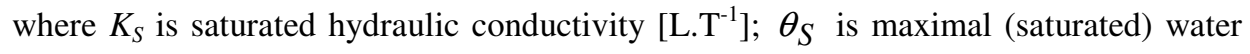
content (equal to the porosity) [-]; $q(t)$ is time dependent rainfall $\left[\mathrm{L}^{\mathrm{T}} \mathrm{T}^{-1}\right] ; \alpha$ is the angle formed by the impermeable layer and the horizontal plane [rad]; and $h_{b}$ is the solution of the Boussinesq equation [L],- this is the vertical distance between the impermeable layer and the groundwater table as it is shown in Fig. 1. 


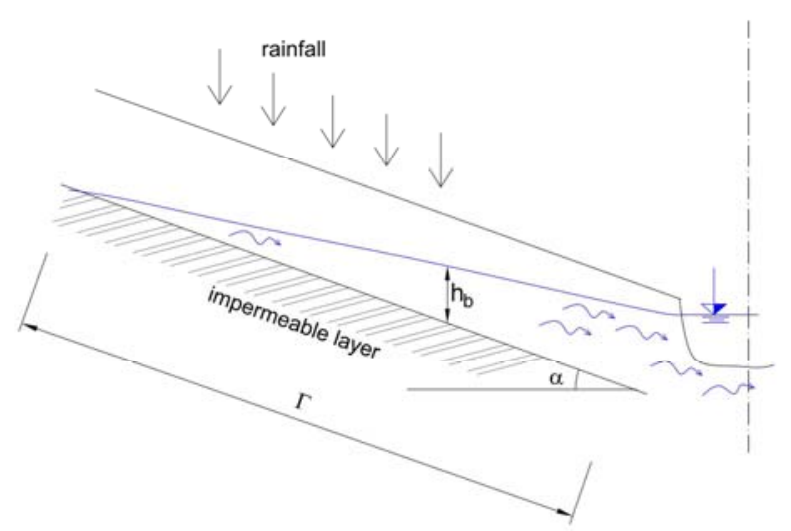

Fig. 1. Scheme of the Boussinesq equation concept. The solution of the Boussinesq equation is referred to as $h_{b}$

\section{Numerical treatment}

This section briefly presents the numerical implementation of the governing equation. A few remarks on adaptive domain decomposition ( $d d$-adaptivity) will be given here, and an interested reader can find further details in the references [16], [17], [18].

Both the Richards and the Boussinesq equation were implemented into the DRUtES project [27] - an object oriented library for finite element method and Schwarz type domain decomposition for solving nonlinear time-dependent convection-difussionreaction type equations. This project was developed by the authors.

The Richards equation has already been the subject of a number of previous publications. The strong and weak formulations of the equation have been covered in depth in [14], [16], [28]. For particular details about finite element method implementation for this class of problems see e.g. [29] or [30].

The Boussinesq equation (5) was assumed in the following scheme

$$
\overbrace{\frac{\theta_{S}}{\cos \alpha}}^{\text {elasticity }} \frac{\partial h_{b}}{\partial t}=\frac{\partial}{\partial x}(\underbrace{K_{S} h_{b}}_{\text {diffusion }} \frac{\partial h_{b}}{\partial x})-\overbrace{\tan \alpha\left(K_{S}-q(t)\right)}^{\text {convection }} \frac{\partial h_{b}}{\partial x}-\underbrace{(-q(t))}_{0^{\text {th }}} \underbrace{(-q,}_{\text {order reaction }},
$$

which satisfies the nonlinear convection-diffusion-reaction type equation.

Since $d d$-adaptivity is not a standard term, the following section will briefly present the method. 


\section{1. dd-adaptivity}

The algorithm referred to here as $d d$-adaptivity is assumed in the following way:

- The mesh is initially decomposed into a large number of small subdomains. This subdomain split is referred as a coarse mesh here;

- Each element of the coarse mesh is referred as a cluster here ;

- A graph of the coarse mesh is created, in order to easily identify neighbors of each cluster;

- Either a single cluster can form a subdomain, or several clusters can be joined to form a subdomain;

- Thus the cluster is the basic unit that is used to form the subdomain split, which changes adaptively in time. The following text will define the rules applied for constructing the subdomains.

Adaptive subdomain construction will be explained on the basis of the following example.

Let us consider a certain Richards equation problem that is solved on the domain $\Omega \subset \mathfrak{R}^{2}$. The computational domain $\Omega$ is already discretized, and the discretization mesh is already decomposed into clusters. Then the adaptivity criterion for $d d$ adaptivity is the number of iterations required for the nonlinear solver to converge. Let us consider some arbitrary non-linear iteration method that evaluates for each iteration level a vector of corrections c. Then for each cluster $i$ there can define a vector of corrections $\mathbf{c}_{i}$, and thus a progression of overlapping or non-overlapping vectors is obtained (depending on the domain decomposition method). Then the adaptivity is performed as follows:

- if a certain cluster $i$ required more than one iteration to converge, i.e. for the first iteration level for the error $\varepsilon_{i}>\varepsilon_{\max }$, where $\varepsilon_{\max }$ is the nonlinear iteration criterion, then this single cluster forms a subdomain;

- if there are neighborhood clusters $i$ that fulfill the iteration criterion for the first iteration level $\varepsilon_{i}>\varepsilon_{\max }$, then these neighborhood clusters are joined into a subdomain;

- if the subdomain is formed from a single cluster only, then this subdomain is marked as 'critical'. This is the subdomain, where the solution develops actively;

- after successful solution of each time level all 'non-critical' subdomains are checked for the error $\varepsilon_{i}$, if the subdomain is not critical and $\varepsilon_{i}>\varepsilon_{\max }$, then the adaptive decomposer procedure is again called in order to create a new subdomain split.

Fig. 2 depicts the $d d$-adaptivity for infiltration into a rectangular domain. The subdomains on the wetting front are always formed from single clusters only, and thus the dimensions of the submatrices with poor conditioning are kept minimal. 


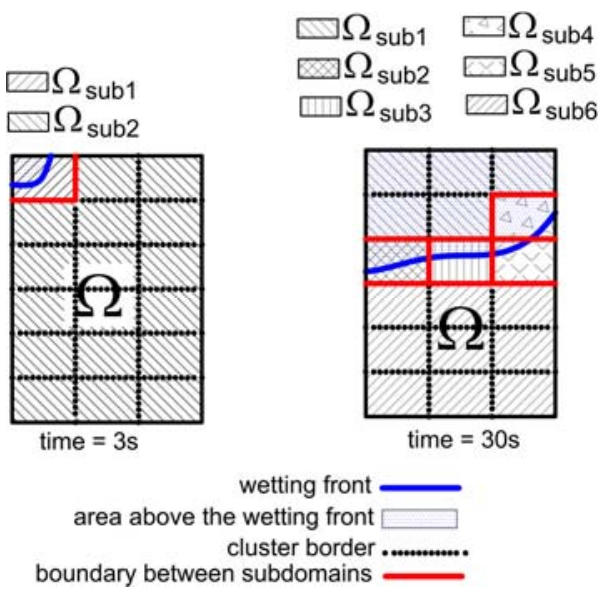

Fig. 2. Sketch of the adaptive domain decomposition algorithm

\subsection{Boussinesq equation estimator}

The Boussinesq equation is just a one-dimensional problem, so its numerical solution has low computational costs. The equation is based on Dupuit's approximation, and its validity is guaranteed for cases of Darcian flow, where the hydraulic gradient does not significantly differ from the geodetic gradient, which is a typical property of groundwater flow. The solution of the Boussinesq equation is used to assemble an initial subdomain split - see Fig. 3, and for estimating the initial condition. The algorithm was constructed in the following way:

- Solve the Boussinesq equation (5) in steady state;

- Map the solution of the Boussinesq equation $\left(h_{b}(x)\right)$ into to the solution of the Richards equation $(h(x, z))$ as follows:

$$
h(x, z)=h_{i m p}(x)+h_{b}(x)-z
$$

for details see Fig. 4, note that above the groundwater table $h(x, z)$ is negative and

$$
\frac{\partial h}{\partial z}=-1, \quad \forall(x, z) \in \Omega,
$$

where $\Omega$ is in this case the computational domain in $\Re^{2}$;

- All clusters below the estimated groundwater water table are joined into a single subdomain (the problem is governed by linear equation (3), the clusters above the groundwater table forms a set of subdomains (the problem is governed by nonlinear equation (1));

- Solve the entire domain with equation (1) with dd-adaptivity. 


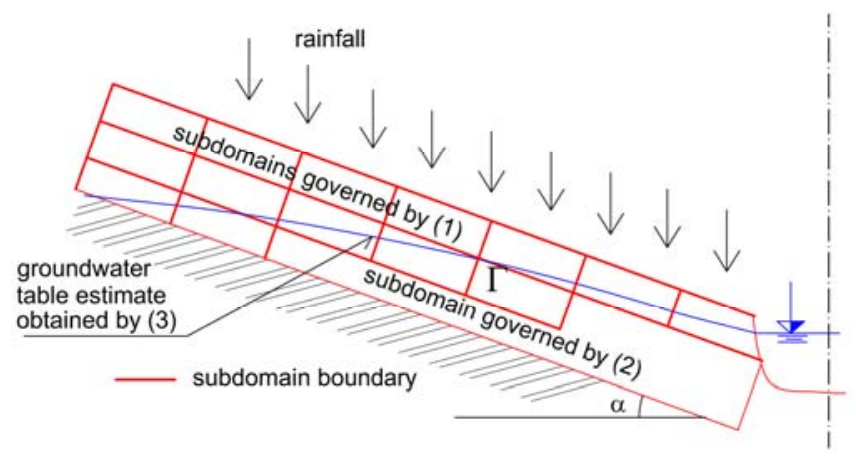

Fig. 3. Subdomain split for dd-adaptivity considering the solution of the Boussinesq equation (5)

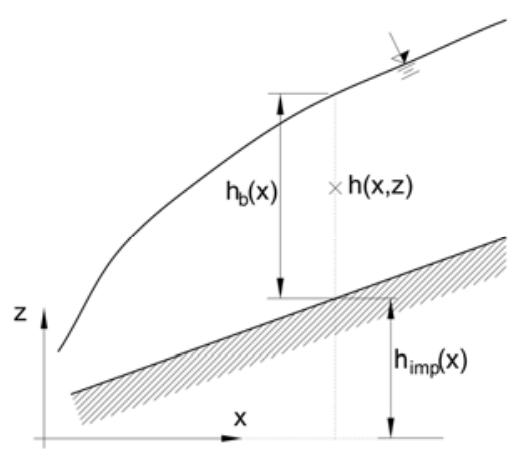

Fig. 4. Scheme of mapping of the Boussinesq equation (5) solution $\left(h_{b}\right)$ into the Richards equation (1) initial condition $(h)$

\section{Two-dimensional hydrological conceptual model of the Modrava catchment}

The Modrava 2 experimental catchment, located in the Sumava mountains, Czech Republic (GPS $48^{\circ} 58^{\prime} 10.489^{\prime \prime} \mathrm{N}, 13^{\circ} 30^{\prime} 25.751^{\prime \prime E}$ ), was considered here for the case study to evaluate runoff model on a real data set.

The Modrava 2 experimental hydrological catchment has the following geometrical properties: the total area is $0.16 \mathrm{~km}^{2}$, the average depth of the impermeable layer is $3 \mathrm{~m}$, and the average slope is 0.15 . The topography of the Modrava catchment is depicted in Fig. 5. If the dimensions of the Modrava catchment is taken into consideration, then appropriate discretization (for an estimated spatial step of $10 \mathrm{~cm}$ ) for $p=1$ approximation would roughly require $3.2 \times 10^{8}$ degrees of freedom (DOFs). Since the Richards equation is nonlinear and requires typically short time steps (for the material involved here the appropriate time step was roughly estimated as $\Delta t=10^{-4} \mathrm{hrs}$ (using the time adaptivity method published by the author in [9], [10]). Since the proposed simulation time covers 
the entire hydrological season (May-October), i.e. 184 days (4416 hrs)), then if using the standard Rothe method for temporal integration there is needed to solve $4 \times 10^{7}$ systems of nonlinear equation sequentially. Since each time level requires for the porous material involved here on an average 5 iterations, the problem is described by $2 \times 10^{8}$ systems of linear equations with $3.2 \times 10^{8}$ DOFs. Therefore several simplifications have to be introduced in order to be able to resolve this problem.

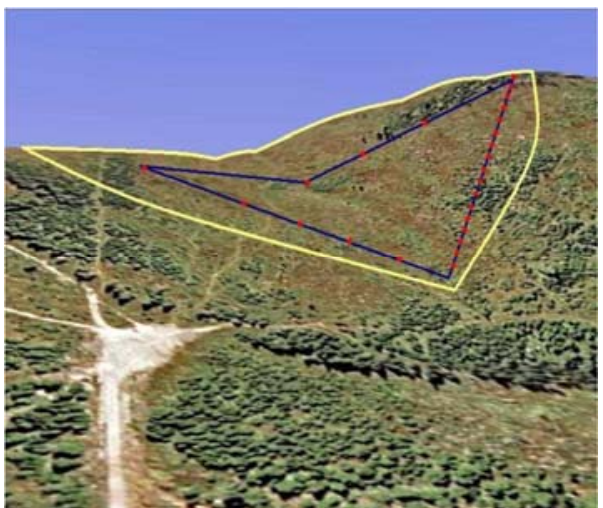

Fig. 5. Modrava 2 catchment topography. The yellow line is the watershed, the blue line depicts the snow measurent stations

\subsection{Transforming the catchment into a two-dimensional conceptual model - hypothesis and model construction}

The following hypothesis was applied here. The flow in the catchment is mainly governed by the subsurface flow. If an appropriate longitudinal cross section is selected then the discharge in the watershed outlet has a linear relation with the specific flux through the lower boundary of this cross section representing the interface between groundwater aquifer and river basin.

Let $q_{S}\left[\mathrm{M} . \mathrm{L}^{-2}\right]$ be a specific discharge (outflow) from two-dimensional computational domain $\Omega$ through the Dirichlet boundary (representing the interface between groundwater aquifer and river basin), and let Q $\left[\mathrm{M} . \mathrm{L}^{-3}\right]$ be a discharge through the closure profile (watershed outlet), then the relation between $q_{S}$ and $Q$ can be expressed as

$$
Q(t)=c_{1} q_{S}(t)+C_{2}
$$

where constants $c_{1}$ and $c_{2}$ are the constants of the linear relation, and express the geometrical properties of the catchment. Moreover constant $c_{2}$ represents the underchannel flow under the watershed outlet (the non measurable part of the catchment runoff).

The selected representative longitudinal cross section is depicted in Fig. 6. The selection was based on the recent georadar observations, which gave a detailed description of the depth and slope of the impermeable layer. 


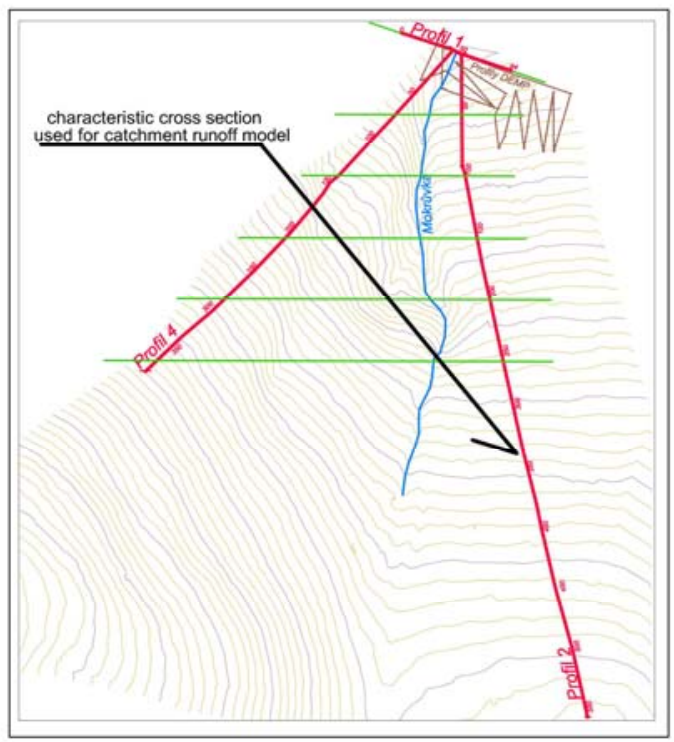

Fig. 6. Characteristic longitudinal cross section of the Modrava 2 catchment

With this simplification, it was possible to construct a computational domain that could be discretized with a significantly lower number DOFs. The simplified domain $\Omega \in \mathfrak{R}^{2}$ was recently discretized with just 36000 DOFs. The domain is extremely narrow, see Fig. 7. The ratio between width and length is only 0.003 , and this geometrical property will affect the conditioning of the system of linear equations that will arise from discretization of this domain.

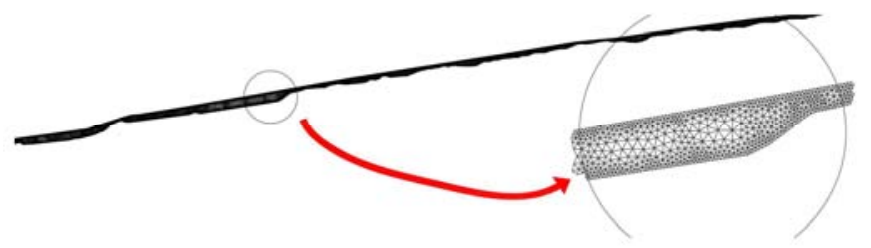

Fig. 7. Discretization of the characteristic longitudinal cross section

\subsection{Model definition - initial and boundary conditions}

As stated above the computational domain $\Omega$ was discretized with a triangular nonuniform mesh with 36053 nodes and 64477 elements (see Fig. 8). 


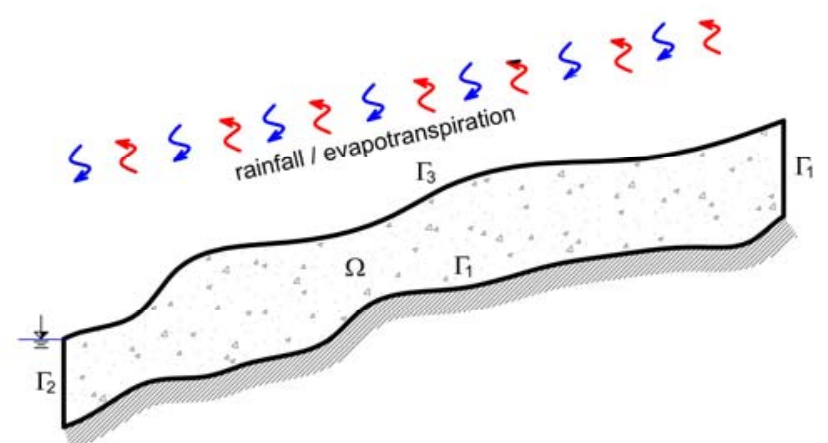

Fig. 8. Scheme of the computational domain $\Omega$ bounded by $\Gamma_{i}$, where $i=1,2,3$

The boundary conditions are stated as follows:

- $\quad \Gamma_{1}$ : Neumann boundary condition (no flow)

$$
\frac{\partial h(\boldsymbol{x}, t)}{\partial \boldsymbol{n}}+n_{2}=0, \quad \forall \vec{x} \in \Gamma_{1} \times[0, T)
$$

where $\boldsymbol{n}$ is the normal vector of the $\Gamma_{1}$ boundary, and $n_{2}$ is the vertical component of the normal vector;

- $\Gamma_{2}$ : Dirichlet boundary condition (water table)

$$
h(\boldsymbol{x}, t)=z_{w}-x_{2}, \quad \forall \boldsymbol{x} \in \Gamma_{2} \times[0, T)
$$

where $z_{w}$ is the elevation of the water table, and $x_{2}$ is the vertical component of each boundary point. In this case $z_{w}$ is located at the interface between the boundary $\Gamma_{2}$ and $\Gamma_{3}$;

- $\Gamma_{3}$ : Neumann boundary condition (rainfall or evapotranspiration)

$$
\frac{\partial h(\boldsymbol{x}, t)}{\partial \boldsymbol{n}}+n_{2}=q(t), \quad \forall \boldsymbol{x} \in \Gamma_{3} \times[0, T),
$$

where $q(t)$ is either the interception or the actual evapotranspiration. The evaluation of the actual evapotranspiration will be explained in the following section.

\section{Evaluating rainfall/evapotranspiration boundary}

The Modrava 2 catchment has a long history of hydrological measurements. The rainfall data and also the discharge data in the watershed outlet are available in 15 min time steps. The problem typically arises with the actual evapotranspiration. Since temperature data are also available, the method recommended by Oudin [31] was 
preffered here. As input data, the method uses only the average daily temperature. The daily potential evapotranspiration amount is then obtained as follows.

$$
\begin{aligned}
& P E=\frac{0.408 R_{e}(T+5)}{100}, \text { if } T>-5, \\
& P E=0, \quad \text { if } T \leq-5,
\end{aligned}
$$

where $P E$ is the daily potential evapotranspiration amount $[\mathrm{mm}] ; T$ is the temperature $\left[{ }^{\circ} \mathrm{C}\right] ; R_{e}$ is the extraterrestrial solar radiation $\left[\mathrm{MJm}^{-2} \mathrm{~d}^{-1}\right]$ evaluated as

$$
R_{e}=\frac{G_{S C} d_{r}}{\pi}\left(\omega_{S} \sin \phi \cos \delta+\cos \phi \cos \delta \sin \omega_{S}\right)
$$

where $G_{S C}$ is the solar constant $118.08\left[\mathrm{MJm}^{-2} \mathrm{~d}^{-1}\right], d_{r}$ is the inverse relative distance Earth-Sun evaluated as

$$
d_{r}=1+0.033 \cos \left(\frac{2 \pi}{365} J\right)
$$

where $J$ is the number of the current day in the year (January $1: J=1$ ), $\delta$ is the solar declination constant evaluated as

$$
\delta=0.409 \sin \left(\frac{2 \pi}{365} J-1.39\right)
$$

$\omega_{S}$ is the sunset hour angle [rad], evaluated as

$$
\omega_{S}=\arccos (-\tan \phi \tan \delta)
$$

and $\phi$ is latitude [rad].

The actual evapotranspiration $A E$ is obtained as follows. If the rainfall intensity is greater than the potential evapotranspiration, then the actual evapotranspiration is equal to the potential evapotranspiration. If the rainfall intensity $r(t)$ is smaller than the potential evapotranspiration $P E$, then the actual evapotranspiration $A E$ is obtained by reducing the potential evapotranspiration $P E$ by the surface water content considered as $\sqrt[3]{\theta(h)^{2}}$, where $\theta(h)$ is the volumetric water content of the surface layer. The volumetric water content is obtained from the solution of the Richards equation (1). Thus the boundary flux states as

$$
q(t)=\left\{\begin{array}{llc}
r(t)-P E(t), & \text { if } \quad r(t)-P E(t) \geq 0, \\
r(t)-P E(t) \theta(h)^{2 / 3}, & \text { if } \quad r(t)-P E(t)<0 .
\end{array}\right.
$$




\section{Initial condition}

Ideally, the initial condition would be the steady state solution of the Richards equation (1), with the boundary condition setup defined in the previous section. Only the time-dependent boundary condition $\Gamma_{3}$ should be assumed as an average value over the measured data of $q(t)$. The total number degrees of freedom is 36053 , and the Dirichlet boundary condition is defined only for 26 nodes. The estimated minimal and maximal eigen numbers are as follows $\lambda_{\min }=-2.1 \times 10^{-14}$ and $\lambda_{\max }=-8.6 \times 10^{-8}$, so the conditioning $\kappa \sim 4 \times 10^{7}$. Despite the fact that this is not an extremely poor conditioning we still preferred to use the Boussinesq equation (5) to assign the initial condition as described in the previous section.

Therefore it was found that it is helpful here to assign the steady state solution to the Boussinesq equation, as explained in section 3.2.

\subsection{Porous media parameters}

The search for parameters of the porous media that describe the catchment is given in a different paper by the authors [32]. For simpicity a homogeneous medium with the parameters presented in Table I.

Table I

The unsaturated hydraulic parameters for the soil profile of the Modrava catchment

\begin{tabular}{|c|r|r|r|r|r|r|}
\hline$K_{S}\left[\mathrm{~m}_{\mathrm{hrs}}{ }^{-1}\right]$ & $\theta_{r}[-]$ & $\theta_{S}[-]$ & $\alpha_{v g}\left[\mathrm{~m}^{-1}\right]$ & $n_{v g}[-$ & $m_{v g}[-$ & $S_{S}\left[\mathrm{~m}^{-1}\right]$ \\
\hline 0.01354 & 0.0 & 0.50 & 3.39 & 1.14 & 0.123 & 0.01 \\
\hline
\end{tabular}

\section{Results and discussion}

As it is given in the above hypothesis there is a linear relation between the outflow through the boundary $\Gamma_{2}$ and the discharge in the watershed outlet. The relation between $q_{S}$ and $Q$ presumed in equation (9) was finally identified as

$$
Q(t)=7962.0 q_{S}(t)-49.0
$$

Fig. 9 depicts the real measured data and the simulated data of discharge in the watershed outlet. It is apparent that during the first $1500 \mathrm{hrs}(\approx 2$ month $)$ of simulation model underestimated the outlet discharge. The reason could be possibly found in the influence of the initial condition that underestimates the real water balance of catchment after the winter season. The rest of the summer season seams to be described more accurately, however it turns out that the real data are smoother than the simulated data. 


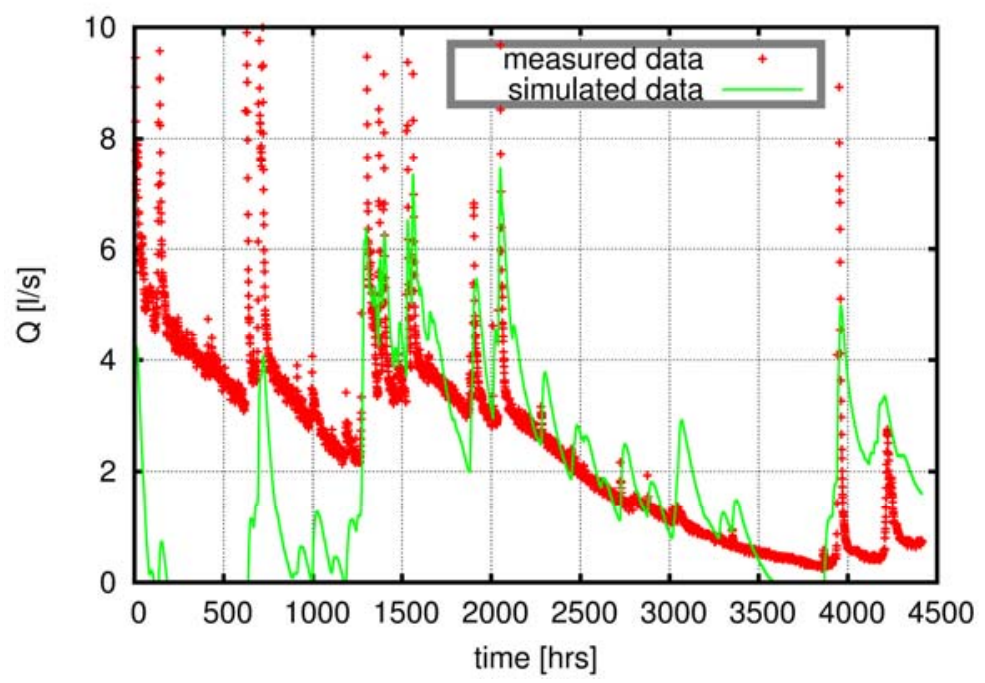

Fig. 9. Plot of real measured data for the discharge in the watershed outlet and simulated data for summer season 2009

\section{Conclusions}

This paper presented deterministic runoff model applied on Modrava 2 catchment, located in Sumava mountains, Czech Republic. The model is based on Richards equation - a mathematical model of flow in porous media under variably saturated conditions. Complicated three-dimensional geometry of the catchment was reduced into two-dimensional conceptual model, where the two-dimensional model geometry originated from representative longitudinal cross section, which was previously well described by georadar observations. Because the Richards equation in saturated state degenerates into Laplace equation, the computational cost could be further reduced by applying the $d d$-adaptivity algorithm. Initial condition was presumed as steady state solution of the Richards equation where the time-dependent rainfall/evapotranspiration was substituted by annual average value. Since the $d d$-adaptivity algorithm cannot be used for steady state solution, and since the conditioning of the matrix resulting from discretization of the steady state model was poor, the initial condition was estimated from the steady state solution of the one-dimensional Boussinesq equation.

The model was evaluated on real hydrological data of Modrava catchment. A technique for evaluating actual evapotranspiration from potential evapotranspiration have been introduced by reducing the potential evapotranspiration by surface water content.

The simulated data were compared with the real measured data. In the first third of the simulated period underestimation of the watershed outlet discharge have been observed. The reason could be probably found in underestimation of the initial catchment water balance. But in the last two thirds of the simulated period the model 
had reasonable correspondence with the real data. However the selection of the representative cross section is still a chalenging topic. Just a single representative cross section is probably not sufficient for representing the entire catchment.

\section{Acknowledgement}

Financial support from the Czech Science Foundation (research project GACR 1311977P) and the financial support from the Technology Agency of the Czech Republic (project TA02021249) is gratefully acknowledged.

\section{References}

[1] Whipkey R. Z. Subsurface storm-flow from forested slopes, International Association of Scientific Hydrology, Vol. 10, No. 2, 1965, pp. 74-85.

[2] Fletcher P. W. The hydrologic function of forest soil in watershed management, Journal of Forestry, Vol. 50, 1952, pp. 359-362.

[3] Freeze R. A. Three-dimensional, transient, Saturated-unsaturated flow in a groundwater basin, Water Resources Research, Vol. 7, No. 2, 1971, pp. 347-366.

[4] Kao C., Bouarfa S., Zimmer D. Steady state analysis of unsaturated flow above a shallow water-table aquifer drained by ditches, Journal of Hydrology, Vol. 250, No. 4, 2001, pp. 122-133.

[5] Kosugi K., Uchida T., Mizuyama T. Numerical calculation of soil pipe flow and its effect on water dynamics in a slope, Hydrological Processes, Vol. 18, No. 4, 2004, pp. 777-789.

[6] Tani M. Analysis of runoff storage relationships to evaluate the runoff-buffering potential of a sloping permeable domain, Journal of Hydrology, Vol. 360, No. 4, 2008, pp. 132-146.

[7] Loritz R., Hassler S. K., Jackisch C., Allrogen N., Schaik L., Wienhöfer J., Zehe E. Picturing and modelling catchments by representative hillslopes, Hydrol. Earth Syst. Sci. Discuss, (in press)

[8] Bishop J. M., Callaghan M. V., Cey E. E., Bentley L. R. Measurement and simulation of subsurface tracer migration to tile drains in low permeability, macroporous soil, Water Resour. Res, Vol. 51, No. 6, 2015, pp. 3956-3981.

[9] Wienhöfer J., Zehe, E. Predicting subsurface storm flow response of a forested hillslope the role of connected flow paths, Hydrol. Earth Syst. Sci, Vol. 18, No. 1, 2014, pp. 121-138.

[10] Klaus J., Zehe, E. Modeling rapid flow response of a tile-drained field site using a 2D physically based model, assessment of 'equifinal' model setups, Hydrol. Process, Vol. 24, No. 12, 2010, pp. 1595-1609.

[11] Troch P. A., Paniconi C., van Loon E. E. Hillslope-storage Boussinesq model for subsurface flow and variable source areas along complex hillslopes, 1, Formulation and characteristic response, Water Resources Research, Vol. 39, No. 11, 2003, pp. 1-10.

[12] Paniconi C., Troch P. A., van Loon E. E., Hilberts A. G. L. Hillslope-storage Boussinesq model for subsurface flow and variable source areas along complex hillslopes: 2, Intercomparison with a three-dimensional Richards equation model, Water Resources Research, Vol. 39, No. 11, 2003, pp. 11-17.

[13] Kuraz M., Mayer P., Leps M, Trpkosova D. An adaptive time discretization of the classical and the dual porosity model of Richards' equation, Journal of Computational and Applied Mathematics, Vol. 233, No. 12, 2010, pp. 3167-3177. 
[14] Kuraz M., Mayer P. Algorithms for solving Darcian flow in structured porous media, Acta Polytechnica, Vol. 53, No. 4, 2013, pp. 347-358.

[15] Šolín P., Kuráž M. Solving the nonstationary Richards equation with adaptive hp-FEM, Advances in Water Resources, Vol. 34, No. 9, 2011, pp. 1062-1081.

[16] Kuraz M., Mayer P., Havlicek V., Pech P. Domain decomposition adaptivity for the Richards equation, Computing, Vol. 95, Suppl. 1, 1913, pp. 501-519.

[17] Kuraz M., Mayer P., Pech P. Solving the nonlinear Richards equation model with adaptive domain decomposition, Journal of Computational and Applied Mathematics, Vol. 270, 2014, pp. 2-14.

[18] Kuraz M., Mayer P., Pech P. Solving the nonlinear and nonstationary Richards equation with multilevel adaptive domain decomposition (dd adaptivity), Applied Mathematics and Computation, Vol. 267, No. C, 2015, pp. 207-222.

[19] Richards L. A. Capillary conduction of liquids through porous mediums, Journal of Applied Physics, Vol. 1, No. 5, 1931, pp. 318-334.

[20] Huyakorn P. S., Thomas S. D., Thompson B. M. Techniques for making finite elements competitive in modeling flow in variably saturated porous media, Water Resources Research, Vol. 20, No. 8, 1984, pp. 1099-1115.

[21] Neuman S. P., Witherspoon P. A. Finite element method analyzing steady seepage with a free surface, Water Resources Research, Vol. 6, No. 3, 1970, pp. 889-897.

[22] Tocci M. D., Kelley C. T., Miller C. T. Accurate and economical solution of the pressure head form of the Richards equation by the method of lines, Advances in Water Resources, Vol. 20, No. 1, 1997, pp. 1-14.

[23] van Genuchten M. T. H. A closed-form equation for predicting the hydraulic conductivity of unsaturated soils, Journal of Soil Science, Vol. 44, No. 5, 1980, pp. 892-898.

[24] Mualem Y. A new model for predicting the hydraulic conductivity of unsaturated porous media, Water Resources Research, Vol. 12, No. 3, 1976, 513-522.

[25] Wooding R. A., Chapman T. G. Groundwater flow over a sloping impermeable layer, Journal of Geophysical Research, Vol. 71, No. 12, 1966. pp. 2895-2902.

[26] Hartani T., Zimmer D., Lesaffre B. Drainage of sloping lands with variable recharge: analytical formulas and model development, Journal of Irrigation and Drainage Engineering, Vol. 127, No. 1, 2001, pp. 8-15.

[27] Kuraz M., Mayer P. DRUtES - an open source library for coupled nonlinear convectiondiffusion-reaction problems, http://www.drutes.org, (last visited 1 March 2016).

[28] Kuraz M., Mayer P., Havlicek V., Pech P., Pavlasek J. Dual permeability variably saturated flow and contaminant transport modeling of a nuclear waste repository with capillary barrier protection, Applied Mathematics and Computation, Vol. 219, No. 13, 2013, pp. 7127-7138.

[29] Kruis J., Koudelka T., Krejčí T. Salt transport in porous materials and its efficient computer implementation, Pollack Periodica, Vol. 9, No. 2, 2014, pp. 111-122.

[30] Brož J., Kruis J. Selection strategy for fixing nodes in FETI-DP method, Pollack Periodica, Vol. 5, No. 2, 2010, pp. 57-68.

[31] Oudin L., Hervieu F., Michel C., Perrin C., Andréassian V., Anctil F., Loumagne C. Which potential evapotranspiration input for a lumped rainfall-runoff model? Part 2, Towards a simple and efficient potential evapotranspiration model for rainfall-runoff modeling, Journal of Hydrology, Vol. 303, No. 1-4, 2005, pp. 290-306.

[32] Kuraz M., Havlicek V., Jacka L. Identifying the hydraulic parameters of Modrava catchment, Journal of Hydrology and Hydromechanics, (under review). 